\title{
Random graphs with a fixed maximum degree
}

\author{
Alan Frieze*and Tomasz Tkocz \\ Department of Mathematical Sciences \\ Carnegie Mellon University \\ Pittsburgh PA15217 \\ U.S.A.
}

March 15, 2019

\begin{abstract}
We study the component structure of the random graph $G=G_{n, m, d}$. Here $d=O(1)$ and $G$ is sampled uniformly from $\mathcal{G}_{n, m, d}$, the set of graphs with vertex set $[n], m$ edges and maximum degree at most $d$. If $m=\mu n / 2$ then we establish a threshold value $\mu_{\star}$ such that if $\mu<\mu_{\star}$ then w.h.p. the maximum component size is $O(\log n)$. If $\mu>\mu_{\star}$ then w.h.p. there is a unique giant component of order $n$ and the remaining components have size $O(\log n)$.
\end{abstract}

2010 Mathematics Subject Classification. 05C80.

Key words. Random Graphs, Maximum Degree.

\section{Introduction}

We study the evolution of the component structure of the random graph $G_{n, m, d}$. Here $d=O(1)$ and $G$ is sampled uniformly from $\mathcal{G}_{n, m, d}$, the set of graphs with vertex set $[n], m$ edges and maximum degree at most $d$. In the past the first author has studied properties of sparse random graphs with a lower bound on minimum degree, see for example [6]. In this paper we study sparse random graphs with a bound on the maximum degree. The model we study is close to, but distinct from that studied by Alon, Benjamini and Stacey [1] and Nachmias and Peres [12. They studied the following model: begin with a random $d$-regular graph and then delete edges with probability $1-p$. They show in [1] that for $d \geq 3$ there is a critical probability $p_{c}=\frac{1}{d-1}$ such that w.h.p. there is a "double jump" from components of maximum size $O(\log n)$ for $p<p_{c}$, a unique giant for $p>p_{c}$ and a mximum component size of order $n^{2 / 3}$ for $p=p_{c}$. The paper [12] does a detailed analysis of the scaling window around $p=p_{c}$.

${ }^{*}$ Research supported in part by NSF grant DMS1661063 
Naively, one might think that this analysis covers $G_{n, m, d}$. We shall see however that $G_{n, m, d}$ and random subgraphs of random regular graphs have distinct degree sequence distributions. In the latter the number of vertices of degree $i=0,1,2, \ldots, d$ will be $n$ times a binomial random variable, whereas in $G_{n, m, d}$ this number will be asymptotic to $n$ times a Poisson random variable, truncated from above.

We will write that $A_{n} \approx B_{n}$ if $A_{n}=(1+o(1)) B_{n}$ and $A_{n} \lesssim B_{n}$ if $A_{n} \leq$ $(1+o(1)) B_{n}$ as $n \rightarrow \infty$.

For $d \geq 1$ and $\lambda>0$ define

$$
s_{d}(\lambda)=\sum_{j=0}^{d} \frac{\lambda^{j}}{j !} \quad \text { and } \quad f_{d}(\lambda)=\lambda \frac{s_{d-1}(\lambda)}{s_{d}(\lambda)} .
$$

Theorem 1. Let $d \geq 2$ and $\mu \in(0, d)$. Let $m=\left\lceil\frac{\mu n}{2}\right\rceil$. Let $G=G_{n, m, d}$ be a random graph chosen uniformly at random from the graphs with $n$ vertices, $m$ edges and maximum degree at most $d$. Let

$$
\mu_{\star}(d)=f_{d}\left(f_{d-1}^{-1}(1)\right), \quad \text { functional inverse being used here, }
$$

where the functions $f_{k}$ are defined in (10) and let $\lambda$ satisfy

$$
f_{d}(\lambda)=\mu \text {. }
$$

The following hold w.h.p.

(a) The number $\nu_{i}, i=0,1, \ldots, d$ of vertices of degree $i$ in $G$ satisfies

$$
\nu_{i} \approx \lambda_{i} n \text { where } \lambda_{i}=\frac{1}{s_{d}(\lambda)} \frac{\lambda^{i}}{i !} .
$$

(b) If $\mu<\mu_{\star}(d)$, then $G$ has all components of size $O(\log n)$.

(c) If $\mu>\mu_{\star}(d)$, then $G$ has a unique giant component of linear size $\Theta n$, where $\Theta$ is defined as follows: let $D=\sum_{i=1}^{L} i \lambda_{i}$ and

$$
g(x)=D-2 x-\sum_{i=1}^{L} i \lambda_{i}\left(1-\frac{2 x}{D}\right)^{i / 2} .
$$

Let $\psi$ be the smallest positive solution to $g(x)=0$. Then

$$
\Theta=1-\sum_{i=1}^{L} \lambda_{i}\left(1-\frac{2 \psi}{D}\right)^{i / 2}
$$

All the other components are of size $O(\log n)$.

Remark 2. Numerical values of the threshold point $\mu_{\star}(d)$ for the average degree for small values of $d$ are gathered in Table 1. Note that we have an exact expression for the case $d=3$. We use $f_{2}(\lambda)=\frac{\lambda(1+\lambda)}{1+\lambda+\lambda^{2} / 2}$ to see that $f_{2}^{-1}(1)=\sqrt{2}$. And then $\mu_{\star}(3)=\frac{\lambda\left(1+\lambda+\lambda^{2} / 2\right)}{1+\lambda+\lambda^{2} / 2+\lambda^{3} / 6}=3(\sqrt{2}-1)$. 
Moreover, if we consider large $d$, then we have, as a function of $d$,

$$
\mu_{\star}(d)=1+\frac{1}{e(d-1) !}-\frac{1}{e d !}+O\left(\frac{1}{(d-1) !^{2}}\right) .
$$

Comparing to the percolation model considered in [1] and [12], where $\mu_{\star}(d)=$ $1+\frac{1}{d-1}$, we see that in our model a giant occurs significantly earlier for large $d$. Approximation (5) can be justified as follows. We have

$$
f_{d}(1)=\frac{s_{d-1}(1)}{s_{d}(1)}=1-\frac{1}{d ! s_{d}(1)}=1-\frac{1}{e d !}+O\left(\frac{1}{d !^{2}}\right)
$$

and

$$
f_{d}^{\prime}(1)=\frac{\left(s_{d-1}(1)+s_{d-2}(1)\right) s_{d}(1)-s_{d-1}(1)^{2}}{s_{d}(1)^{2}}=1-\frac{1}{e d !}+O\left(\frac{1}{d !^{2}}\right)
$$

(Express here $s_{d-1}$ and $s_{d-2}$ in terms of $s_{d}$ and use $s_{d}(1)=e-O(1 / d !)$ ).

If $f_{d-1}^{-1}(1)=1+\varepsilon$, then

$$
1=f_{d-1}(1+\varepsilon)=f_{d-1}(1)+f_{d-1}^{\prime}(1) \varepsilon+O\left(\varepsilon^{2}\right),
$$

which gives

$$
\varepsilon+O\left(\varepsilon^{2}\right)=\frac{1-f_{d-1}(1)}{f_{d-1}^{\prime}(1)}=\frac{1}{e(d-1) !}+O\left(\frac{1}{(d-1) ! d !}\right) .
$$

Consequently,

$$
\mu_{\star}(d)=f_{d}(1+\varepsilon)=f_{d}(1)+f_{d}^{\prime}(1) \frac{1-f_{d-1}(1)}{f_{d-1}^{\prime}(1)}+O\left(\varepsilon^{2}\right) .
$$

and (5) follows.

\begin{tabular}{|c|c|}
\hline$d$ & $\mu_{\star}(d)$ \\
\hline 2 & $\infty$ \\
3 & $3(\sqrt{2}-1)=1.23264 \ldots$ \\
4 & 1.05783 \\
5 & 1.01309 \\
6 & 1.00259 \\
7 & 1.00044 \\
8 & 1.00006 \\
\hline
\end{tabular}

Table 1: Numerical values of $\mu_{\star}(d)$ for small $d$.

\section{Proof of Theorem 1}

The main idea is to estimate the degree distribution of $G_{n, m, d}$ and then apply the results of Molloy and Reed [10], [11. 


\subsection{Technical Lemmas}

The following lemmas will be needed for the proof of part (a).

Lemma 3. Let $\lambda>0, d \geq 1$. Let $Z_{1}, Z_{2}, \ldots$ be i.i.d. random variables with

$$
\mathbb{P}\left(Z_{i}=k\right)=c_{\lambda} \frac{\lambda^{k}}{k !}, \quad k=0,1, \ldots, d,
$$

where

$$
c_{\lambda}=\frac{1}{s_{d}(\lambda)}
$$

(a truncated Poisson distribution). Let $\left(x_{1}, \ldots, x_{n}\right)$ be a random vector of occupancies of boxes when $m$ distinguishable balls are placed uniformly at random into $n$ labelled boxes, each with capacity $d$. Then the vector $\left(Z_{1}, \ldots, Z_{n}\right)$ conditioned on $\sum_{j=1}^{n} Z_{j}=m$ has the same distribution as $\left(x_{1}, \ldots, x_{n}\right)$.

Proof. Let $A$ be the set of vectors $z=\left(z_{1}, \ldots, z_{n}\right)$ of non-negative integers $z_{j}$ such that $\sum_{j=1}^{n} z_{j}=m$ and $z_{j} \leq d$ for every $j$. Fix $z \in A$. We have

$$
\begin{aligned}
\mathbb{P}\left(\left(Z_{1}, \ldots, Z_{n}\right)=z \mid \sum_{j=1}^{n} Z_{j}=m\right) & =\frac{\mathbb{P}\left(\left(Z_{1}, \ldots, Z_{n}\right)=z\right)}{\mathbb{P}\left(\sum_{j=1}^{n} Z_{j}=m\right)} \\
& =\frac{\prod_{j=1}^{n} c_{\lambda} \frac{\lambda^{z_{j}}}{z_{j} !}}{\sum_{z \in A} \prod_{j=1}^{n} c_{\lambda} \frac{\lambda^{z_{j}}}{z_{j} !}}=\frac{\frac{1}{z_{1} ! \cdot \ldots \cdot z_{n} !}}{\sum_{z \in A} \frac{1}{z_{1} ! \cdot \ldots \cdot z_{n} !}}
\end{aligned}
$$

On the other hand, there are $\frac{m !}{z_{1} ! \cdots \cdot z_{n} !}$ ways to place $m$ balls into $n$ labelled boxes in such a way that the $j$ th box gets $z_{j}$ balls. Therefore,

$$
\mathbb{P}\left(\left(x_{1}, \ldots, x_{n}\right)=z\right)=\frac{\frac{m !}{z_{1} ! \cdot \ldots \cdot z_{n} !}}{\sum_{z \in A} \frac{m !}{z_{1} ! \cdot \ldots \cdot z_{n} !}}=\mathbb{P}\left(\left(Z_{1}, \ldots, Z_{n}\right)=z \mid \sum_{j=1}^{n} Z_{j}=m\right) .
$$

Remark 4. The same argument can be adapted to different constraints for the occupancies of the boxes. In general, we can replace $k \in\{0,1, \ldots, d\}$ by $k \in I$ for some set of non-negative integers $I$. For example, instead of restricting the maximal occupancy, we can require a minimal occupancy (which has appeared in Lemma 4 in [2]), or that the occupancy is even, etc.

A straightforward consequence of a standard i.i.d. case of the local central limit theorem (see, e.g. Theorem 3.5.2 in [5]) is the following lemma which will help us get rid of the conditioning from Lemma 3 .

Lemma 5. Let $\lambda>0, d \geq 1$. Let $Z_{1}, Z_{2}, \ldots$ be i.i.d. truncated Poisson random variables defined by (6) and (77). Then

$$
\sup _{m=0,1,2, \ldots} \sqrt{n}\left|\mathbb{P}\left(Z_{1}+\ldots+Z_{n}=m\right)-\frac{1}{\sqrt{2 \pi n \sigma^{2}}} \exp \left\{-\frac{(m-\mu n)^{2}}{2 n \sigma^{2}}\right\}\right| \underset{n \rightarrow \infty}{\longrightarrow} 0,
$$

where $\mu=\mathbb{E} Z_{1}$ and $\sigma^{2}=\operatorname{Var}\left(Z_{1}\right)$. 
We shall also need two lemmas concerning the function $s_{d}$ from (1). A function $f$ is $\log$-concave if $\log f$ is concave.

Lemma 6. For every $\lambda>0$, the sequence $\left(s_{d}(\lambda)\right)_{d=0}^{\infty}$ defined by (1) is log-concave, that is $s_{d-1}(\lambda) s_{d+1}(\lambda) \leq s_{d}(\lambda)^{2}, d \geq 1$.

Proof. First note that the product of log-concave functions is log-concave. Integration by parts yields

$$
e^{-\lambda} s_{d}(\lambda)=\int_{\lambda}^{\infty} \frac{t^{d}}{d !} e^{-t} \mathrm{~d} t
$$

Given this integral representation, the log-concavity of $\left(s_{d}(\lambda)\right)_{d=0}^{\infty}$ follows from a more general result saying that if $f:(0, \infty) \rightarrow[0, \infty)$ is log-concave, then the function $(0,+\infty) \ni p \mapsto \int_{0}^{\infty} \frac{t^{p}}{\Gamma(p+1)} f(t) \mathrm{d} t$ is also log-concave (apply to $f(t)=$ $e^{-t} \mathbf{1}_{(\lambda, \infty)}(t)$ ). This result goes back to Borell's work [4] (for this exact formulation see, e.g. Corollary 5.13 in [8] or Theorem 5 in 13 containing a direct proof).

Remark 7 . The above theorem and proof uses two related notions of log-concavity. They are reconciled by the fact that if $f:(0, \infty) \rightarrow[0, \infty)$ is log-concave then the sequence $f(i), i=0,1, \ldots$ is also log-concave.

Lemma 8. For every $k \geq 1$, the function $f_{k}$ is strictly increasing on $(0, \infty)$ and onto $(0, k)$. In particular, the functional inverse, $f_{k}^{-1}:(0, k) \rightarrow(0, \infty)$ is well-defined, also strictly increasing.

Proof. Fix $k \geq 1$ and consider $f_{k}$ : rewriting (9) in terms of the upper incomplete gamma function $\Gamma(s, x)=\int_{x}^{\infty} t^{s-1} e^{-t} \mathrm{~d} t$, we have

$$
f_{k}(x)=k \frac{x \Gamma(k, x)}{\Gamma(k+1, x)} .
$$

Differentiating,

$$
\frac{\Gamma(k+1, x)^{2}}{k} \frac{\mathrm{d}}{\mathrm{d} x} f_{k+1}(x)=\left(\Gamma(k, x)-x^{k} e^{-x}\right) \Gamma(k+1, x)+x^{k+1} e^{-x} \Gamma(k, x) .
$$

Using $\Gamma(k+1, x)=k \Gamma(k, x)+x^{k} e^{-x}$ we can express the condition $\frac{\mathrm{d}}{\mathrm{d} x} f_{k+1}(x)>0$ as a quadratic inequality for $\Gamma(k, x)$ :

$$
k \Gamma(k, x)^{2}+x^{k} e^{-x}(x-k+1) \Gamma(k, x)-x^{2 k} e^{-2 x}>0,
$$

or

$$
\left(\Gamma(k, x)+\frac{x^{k} e^{-x}(x-k+1)}{2 k}\right)^{2}>\frac{x^{2 k} e^{-2 x}}{k}+\left(\frac{x^{k} e^{-x}(x-k+1)}{2 k}\right)^{2}
$$

or

$$
\Gamma(k, x)>\frac{x^{k} e^{-x}}{2 k}\left(\sqrt{(x-k+1)^{2}+4 k}-(x-k+1)\right) .
$$

Let $h(x)$ be the left hand side minus the right hand side of (10). Clearly, $h(0)=$ $(k-1) !>0$. Moreover, using a standard asymptotic expansion

$$
\Gamma(k, x) \approx x^{k-1} e^{-x}\left(1+\frac{k-1}{x}+\frac{(k-1)(k-2)}{x^{2}}+\ldots\right), \text { as } x \rightarrow \infty,
$$


we can check that $h(x) \approx x^{k-1} e^{-x}\left(\frac{1}{x^{2}}+\ldots\right)$, so $h(x) \rightarrow 0$ as $x \rightarrow \infty$. Thus to see that $h(x)>0$ for $x>0$, it suffices to check that $h^{\prime}(x)<0$ for $x>0$. We have,

$$
\begin{aligned}
h^{\prime}(x) & =-x^{k-1} e^{-x}-\frac{x^{k-1} e^{-x}}{2 k}(k-x)\left(\frac{x-k+1}{\sqrt{(x-k+1)^{2}+4 k}}-1\right) \\
& =-\frac{x^{k-1} e^{-x}}{2 k \sqrt{(x-k+1)^{2}+4 k}}\left(2 k \sqrt{(x-k+1)^{2}+4 k}+(k-x)((x-k+1)\right. \\
& \left.=-\frac{x^{k-1} e^{-x}}{2 k \sqrt{(x-k+1)^{2}+4 k}}\left((k+x) \sqrt{(x-k+1)^{2}+4 k}\right)\right)
\end{aligned}
$$

so $h^{\prime}(x)<0$ is equivalent to

$$
(k+x) \sqrt{(x-k+1)^{2}+4 k}>(x-k)(x-k+1) .
$$

When $k-1<x<k$, the right hand side is negative, so the inequality is clearly true. Otherwise, squaring it, we equivalently get

$$
(k+x)^{2}\left((x-k+1)^{2}+4 k\right)>(x-k)^{2}(x-k+1)^{2}
$$

which is clearly true because $(k+x)^{2}>(x-k)^{2}$ for $x>0$.

It is clear from (7) and (11) that $f_{k}$ is a ratio of two polynomials, each of degree $k$ and $f_{k}(x)=\frac{\frac{x^{k}}{(k-1) !}+\ldots}{\frac{x^{k}}{k !}+\ldots}$, so $f_{k}(x) \rightarrow k$ as $x \rightarrow \infty$. This combined with the monotonicity and $f_{k}(0)=0$ justifies that $f_{k}$ is a bijection onto $(0, k)$.

\subsection{Main elements of the proof}

Let $\mathcal{D}$ be the set of all sequences of nonnegative integers $x_{1}, \ldots, x_{n} \leq d$ such that $\sum x_{i}=2 m$ (possible degrees). For $x \in \mathcal{D}$, let $\mathcal{G}_{n, x}$ be the set of all simple graphs on vertex set $[n]$ such that vertex $i$ has degree $x_{i}, i=1,2, \ldots, n$. We study graphs in $\mathcal{G}_{n, x}$ via the Configuration Model of Bollobás [3]. We do this as follows: let $Z_{x}$ be the multi-set consisting of $x_{i}$ copies of $i$, for $i=1,2, \ldots, n$ and let $z=z_{1}, z_{2}, \ldots, z_{2 m}$ be a random permutation of $Z_{x}$. We then define $\Gamma_{z}$ to be the (configuration) multigraph with vertex set $[n]$ and edges $\left\{z_{2 i-1}, z_{2 i}\right\}$ for $i=1,2, \ldots, m$. It is a classical fact that conditional on being simple, $\Gamma_{z}$ is distributed as a uniform random member of $\mathcal{G}_{n, x}$, see for example Section 11.1 of [7].

Let $\alpha_{x}=\frac{\sum_{i} x_{i}\left(x_{i}-1\right)}{2 m}$. Note that $0 \leq \alpha_{x} \leq d$. It is known that

$$
\left|\mathcal{G}_{n, x}\right| \approx e^{-\alpha_{x}\left(\alpha_{x}+1\right)} \frac{(2 m) !}{\prod_{i} x_{i} !}
$$

as $n \rightarrow \infty$ with the $o(1)$ term being uniform in $x$ (in fact, depending only on $\left.\Delta=\max _{i} x_{i}\right)$. Here the term $e^{-\alpha_{x}\left(\alpha_{x}+1\right)}$ is the asymptotic probability that $\Gamma_{z}$ is simple. Therefore, for any $x \in \mathcal{D}$, we have

$$
\mathbb{P}\left(G_{n, m, d} \in \mathcal{G}_{n, x}\right)=\frac{\left|\mathcal{G}_{n, x}\right|}{\sum_{y \in \mathcal{D}}\left|\mathcal{G}_{n, y}\right|} \lesssim e^{d(d+1)} \frac{\frac{(2 m) !}{\prod_{i} x_{i} !}}{\sum_{y \in \mathcal{D}} \frac{(2 m) !}{\prod_{i} y_{i} !}},
$$


which by Lemma 3 gives

$$
\mathbb{P}\left(G_{n, m, d} \in \mathcal{G}_{n, x}\right) \lesssim e^{d(d+1)} \mathbb{P}\left(Z=x \mid \sum_{i} Z_{i}=2 m\right),
$$

where $Z_{1}, \ldots, Z_{n}$ are i.i.d. truncated Poisson random variables defined in (6).

For any graph property $\mathcal{P}$, we thus have

$$
\begin{aligned}
\mathbb{P}\left(G_{n, m, d} \in \mathcal{P}\right) & =\sum_{x \in \mathcal{D}} \mathbb{P}\left(G_{n, m, d} \in \mathcal{P} \mid G_{n, m, d} \in \mathcal{G}_{n, x}\right) \mathbb{P}\left(G_{n, m, d} \in \mathcal{G}_{n, x}\right) \\
& =\sum_{x \in \mathcal{D}} \mathbb{P}\left(G_{n, x} \in \mathcal{P}\right) \mathbb{P}\left(G_{n, m, d} \in \mathcal{G}_{n, x}\right) \\
& \lesssim e^{d(d+1)} \sum_{x \in \mathcal{D}} \mathbb{P}\left(G_{n, x} \in \mathcal{P}\right) \mathbb{P}\left(Z=x \mid \sum_{i} Z_{i}=2 m\right)
\end{aligned}
$$

where $G_{n, x}$ denotes a random graph selected uniformly at random from $\mathcal{G}_{n, x}$.

To handle the conditioning, we have chosen $\lambda$ so that $\mu=\mathbb{E} Z_{1}$, that is the value of $\lambda$ given by (2).

From Lemma 5 we get that for arbitrary $\delta>0$, for sufficiently large $n$,

$$
\mathbb{P}\left(Z_{1}+\ldots+Z_{n}=2 m\right) \geq-\frac{\delta}{\sqrt{n}}+\frac{1}{\sqrt{2 \pi n \sigma^{2}}} \exp \left\{-\frac{(2 m-\mu n)^{2}}{2 n \sigma^{2}}\right\} .
$$

Since $2 m-\mu n=2\left\lceil\frac{\mu n}{2}\right\rceil-\mu n \leq 2$ and $\sigma^{2}=\operatorname{Var}\left(Z_{1}\right)$ depends only on $\lambda$ and $d$, hence only on $\mu$ and $d$, for sufficiently large $n$, the exponential factor is greater than, say $1 / 2$. Adjusting $\delta$ appropriately and using that $\sigma^{2} \leq \mu$, in fact,

$$
\operatorname{Var}\left(Z_{1}\right)=\mathbb{E} Z_{1}\left(Z_{1}-1\right)-\left(\mathbb{E} Z_{1}\right)^{2}+\mathbb{E} Z_{1}=\lambda^{2} \frac{s_{d-2}(\lambda) s_{d}(\lambda)-s_{d-1}(\lambda)^{2}}{s_{d}(\lambda)}+\mathbb{E} Z_{1},
$$

which by Lemma 6 is bounded by $\mathbb{E} Z_{1}=\mu$, we get for sufficiently large $n$,

$$
\mathbb{P}\left(Z_{1}+\ldots+Z_{n}=2 m\right) \geq \frac{1}{10 \sqrt{\mu n}} .
$$

Thus, for every $x \in \mathcal{D}$,

$$
\mathbb{P}\left(Z=x \mid \sum_{i} Z_{i}=2 m\right) \leq \frac{\mathbb{P}(Z=x)}{\mathbb{P}\left(\sum_{i} Z_{i}=2 m\right)} \leq 10 \sqrt{\mu n} \mathbb{P}(Z=x) .
$$

The next step is to break the sum in (11) into likely and unlikely degree sequences. Note that $\mathbb{E} \sum_{j=1}^{d} \mathbf{1}_{\left\{Z_{j}=i\right\}}=n \mathbb{P}\left(Z_{1}=i\right)=n \lambda_{i}$. By Hoeffding's inequality,

$$
\mathbb{P}\left(\left|\sum_{j=1}^{n} \mathbf{1}_{\left\{Z_{j}=i\right\}}-n \lambda_{i}\right|>\varepsilon n \lambda_{i}\right) \leq 2 e^{-\varepsilon^{2} n \lambda_{i} / 3}, \quad \varepsilon>0 .
$$

Put $\varepsilon=n^{-1 / 3} \frac{1}{\max _{i} \lambda_{i}}$. The union bound yields

$$
\mathbb{P}\left(\exists i \leq d\left|\sum_{j=1}^{n} \mathbf{1}_{\left\{Z_{j}=i\right\}}-n \lambda_{i}\right|>n^{2 / 3}\right) \leq 2 d \exp \left\{-n^{1 / 3} \frac{\min _{i} \lambda_{i}}{3\left(\max _{i} \lambda_{i}\right)^{2}}\right\} .
$$


This proves (a). It also shows that w.h.p. $n \lambda_{i}, i=0,1, \ldots, d$ asymptotically defines the degree distribution of $G_{n, m, d}$. Also, given that $x$ is chosen uniformly at random from $\mathcal{D}$, we see that the distribution of $G_{n, x}$ in this case is the same as the distribution of the configuration model for the given degree sequence.

To prove (b) and (c), we will use the Molloy-Reed criterion (see [10, 11] and Theorem 11.11 in 7 for the exact formulation we shall use). First define

$$
\mathcal{A}=\left\{x=\left(x_{1}, \ldots, x_{n}\right) \in \mathcal{D}, \exists i \leq d\left|\sum_{j=1}^{n} \mathbf{1}_{\left\{x_{j}=i\right\}}-n \lambda_{i}\right|>n^{2 / 3}\right\} .
$$

Then, using (13) and (14),

$$
\begin{aligned}
\sum_{x \in \mathcal{A}} \mathbb{P}\left(G_{n, x} \in \mathcal{P}\right) \mathbb{P}\left(Z=x \mid \sum_{i} Z_{i}=2 m\right) & \leq 10 \sqrt{\mu n} \sum_{x \in \mathcal{A}} \mathbb{P}(Z=x) \\
& =10 \sqrt{\mu n} \mathbb{P}(Z \in \mathcal{A}) \\
& \leq 20 d \sqrt{\mu n} \exp \left\{-n^{1 / 3} \frac{\min _{i} \lambda_{i}}{3\left(\max _{i} \lambda_{i}\right)^{2}}\right\} .
\end{aligned}
$$

It remains to handle the typical terms $x \in \mathcal{D} \backslash \mathcal{A}$ in (11). For such $x$, we now estimate $p_{x}=\mathbb{P}\left(G_{n, x} \in \mathcal{P}\right)$ in two cases: for $\mathcal{P}$ being the complement of (i) "there are only small components", and (ii) "there is a giant" depending on the behaviour of the degree sequences.

Let $Q=\sum_{i=0}^{d} i(i-2) \lambda_{i}$. Note that by the definition of $\mathcal{A}$, for every $x \in \mathcal{D} \backslash \mathcal{A}$, the number of vertices in $G_{n, x}$ is $n \lambda_{i}+O\left(n^{2 / 3}\right)$, so it is justified to use the MolloyReed criterion and we obtain that: if $Q<0$, then $\max _{x} p_{x} \rightarrow 0$ in the case (i), and the same if $Q>0$ in the case (ii). Finally note that

$$
Q=\lambda^{2} \frac{s_{d-2}(\lambda)}{s_{d}(\lambda)}-\lambda \frac{s_{d-1}(\lambda)}{s_{d}(\lambda)}=f_{d}(\lambda)\left(f_{d-1}(\lambda)-1\right)
$$

and Lemma 8 together with the definition of $\lambda$, that is (2), finishes the proof. The expression for $\Theta$ is in [11. (One can also find a simplified proof of the Molloy-Reed results in [7], Theorem 11.11.)

\section{Conclusions}

We have found tight expressions for the degree sequence of $G_{n, m, d}$ and we have used the Molloy-Reed results to exploit them. In future work, we plan to study the scaling window around $Q$ close to zero. Hatami and Molloy [9] consider this case and their results show that we can expect a maximum component size close to $n^{2 / 3}$ in this case. They deal with a general degree sequence and perhaps we can prove tighter results for our specific case.

\section{References}

[1] N. Alon, I. Benjamini and A. Stacey, Percolation on finite graphs and isoperimetric inequalities, The Annals of Probability 32 (2004) 1727-1745. 
[2] J. Aronson, A.M. Frieze and B. Pittel, Maximum matchings in sparse random graphs: Karp-Sipser revisited, Random Structures and Algorithms 12 (1998), 111-178.

[3] B. Bollobás, A probabilistic proof of an asymptotic formula for the number of labelled graphs, European Journal on Combinatorics 1 (1980) 311-316.

[4] C. Borell, Complements of Lyapunov's inequality., Math. Ann. 205 (1973) 323331.

[5] R. Durrett, Probability: theory and examples, Fourth edition, Cambridge Series in Statistical and Probabilistic Mathematics, 31. Cambridge University Press, Cambridge, 2010.

[6] A.M. Frieze, On a Greedy 2-Matching Algorithm and Hamilton Cycles in Random Graphs with Minimum Degree at Least Three, Random structures and Algorithms 45 (2014) 443-497.

[7] A.M. Frieze and M. Karoński, Introduction to random graphs. Cambridge University Press, Cambridge, 2016.

[8] O. Guédon, P. Nayar and T. Tkocz, Concentration inequalities and geometry of convex bodies, Analytical and probabilistic methods in the geometry of convex bodies, 9-86, IMPAN Lect. Notes, 2, Polish Acad. Sci. Inst. Math., Warsaw, 2014.

[9] H. Hatami and M. Molloy. The scaling window for a random graph with a given degree sequence, Random Structures and Algorithms 41 (2012) 99-123.

[10] M. Molloy and B. Reed, A critical point for random graphs with a given degree sequence. Random Structures Algorithms 6 (1995) 161-179.

[11] M. Molloy and B. Reed, The size of the giant component of a random graph with a given degree sequence, Combinatorics. Probability and Computing 7 (1998) 295-305.

[12] A. Nachmias and Y. Peres, Critical percolation on random regular graphs, Random Structures and Algorithms 36 (2010) 111-148.

[13] P. Nayar and K. Oleszkiewicz, Khinchine type inequalities with optimal constants via ultra log-concavity, Positivity 16 (2012) 359-371. 